Copyright ACM, 2014. This is the author's version of the work. It is posted here by permission of ACM for your personal use. Not for redistribution. The definitive version was published in Proceedings of the 29th ACM/IEEE international conference on Automated software engineering, ISBN: 978-1-4503-3013-8, http://dx.doi.org/10.1145/2642937.2642945.

\title{
Formalisation of the Integration of Behavior Trees
}

\author{
Kushal Ahmed, M. A. Hakim Newton, Lian Wen, Abdul Sattar \\ Institute for Integrated and Intelligent Systems \\ Griffith University, Nathan, QLD 4111, Australia \\ \{k.ahmed, hakim.newton, I.wen, a.sattar\}@griffith.edu.au
}

\begin{abstract}
In this paper, we present a formal definition of the integration of the requirements modeling language Behavior Trees (BTs). We first provide the semantic integration of two interrelated BTs using an extended version of Communicating Sequential Processes. We then use a Semantic Network Model to capture a set of interrelated BTs, and develop algorithm to integrate them all into one BT. This formalisation facilitates developing (semi-)automated tools for modeling the requirements of large-scale software intensive systems.
\end{abstract}

\section{Categories and Subject Descriptors}

D.2.1 [Software Engineering]: Requirements/Specificationsrequirements modeling, process automation

\section{Keywords}

Behavior Trees; Behavior Engineering; Integration; Merge; CSP; Semantic Network Model

\section{INTRODUCTION}

Requirements Engineering (RE) often starts with a set of scattered and unstructured system requirements with a view to achieving an integrated and structured formal specification. Unsurprisingly, such requirements may be filled with problems, such as ambiguity, inconsistency, redundancy, and incompleteness. As a result, the process of transforming the requirements into a formal specification is extremely complicated. The transformation process must therefore be able to identify issues in the requirements in a way easy to understand, and the model must be structured such that it can be cross referenced with the original requirements document.

Behavior Engineering (BE) was developed to address this problem $[5,15]$. It uses Behavior Tree (BT) as the graphical notation. A BT contains a range of constructs that cover state-based manipulations, as well as more abstract concepts such as synchronisation and message passing, along with

Permission to make digital or hard copies of all or part of this work for personal or classroom use is granted without fee provided that copies are not made or distributed for profit or commercial advantage and that copies bear this notice and the full citation on the first page. Copyrights for components of this work owned by others than ACM must be honored. Abstracting with credit is permitted. To copy otherwise, or republish, to post on servers or to redistribute to lists, requires prior specific permission and/or a fee. Request permissions from permissions@acm.org.

ASE'14, September 15-19, 2014, Vasteras, Sweden.

Copyright 2014 ACM 978-1-4503-3013-8/14/09 ...\$15.00.

http://dx.doi.org/10.1145/2642937.2642945. typical concurrency, choice and iteration control structures familiar to specification and programming languages.

Each requirement is translated into one or more BTs, and each node in the tree is tagged with the requirements identifier allowing traceability back to the original informal requirements. BTs may have syntactically matching constructs. Therefore, they may then be progressively integrated into one holistic model of the system, which serves as a formal specification. This process may reveal inconsistencies, redundancies, incompleteness, and ambiguities. BTs have been adopted for industrial use, in particular Raytheon Australia [1], who invested resources to developing a BT editor [10]. There are many other tools on BTs $[14,18,20,23]$, some of them support validation and verification as well.

However, the integration of the BTs is still informal, adhoc and manual. Colvin and Hayes [4] provided the semantics of a BT using Hoare's process algebra Communicating Sequential Processes (CSP) $[9,19]$ as its base, which is a well established and elegant formal notation for describing interactions between concurrent processes. By extending it, they defined $\mathrm{CSP}_{\sigma}$ to include state-based constructs such as tests and updates, and message passing facility similar to publish/subscribe models of the communication [6]. In this paper, we first provide the semantic integration of two interrelated BTs using an extended version of $\mathrm{CSP}_{\sigma}$. We then use a Semantic Network Model (SNM) [3] to capture a set of interrelated BTs, and develop algorithm to integrate them all into one BT. This formalisation facilitates developing (semi-)automated tools for modeling the requirements of large-scale software intensive systems.

Paper organisation: Section 2 briefly describes BTs and their interrelations, $\mathrm{CSP}_{\sigma}$ and SNM; Section 3 formalises the integration of two interrelated BTs; Section 4 extends the formalism for a set of BTs; Section 5 explores related literature; and finally Section 6 presents our conclusion.

\section{BACKGROUND}

In this section, we briefly describe the constructs of BTs and their interrelations, $\mathrm{CSP}_{\sigma}$ and SNM.

BTs and their interrelations: Fig. 1(a) shows a BT of a requirement (ID: R2) of a Security Alarm System (SAS) $[3,17]$. It mentions the initialisation scenarios for activating the SAS. Fig. 1(b) displays the contents of a BT node. Each node is associated with a component having a behavior. The type of behavior may be internal input $(><)$, external input $(\gg \ll)$, internal output $(<>)$, external output $(\ll \gg)$, event (????), selection, (??), or state realisation ([]) etc. 


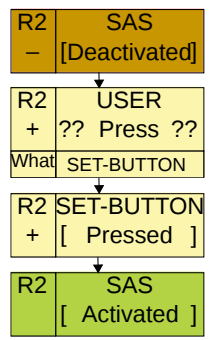

(e) traceability (a) component (f) node operator link

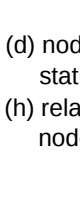
name (g) node R4 ALÁRM | |b| label status $\rightarrow+$ [ Emitted ] (c) behavior \begin{tabular}{l|c|c} 
related What & TONE & (b) behavio
\end{tabular}

(b) Attributes of a node

(a) BT for R2 (BT2)

Figure 1: Behavior Tree and Attributes of a node

The node operators such as reversion $(\wedge)$, reference $(=>)$, branch kill $(--)$ and synchronisation $(=)$ are used to indicate control flow beyond simple sequential execution and branching. The nodes can be connected in different ways such as sequential, parallel, atomic and alternative $([4,5])$.

Notice BT2 in Fig. 1 (a) and BT3 (ID: R3) in Fig. 2(a). BT2's ending scenario activating the SAS is the starting scenario of BT3. This means, BT2 and BT3 are interrelated, and can be integrated into one BT (Fig. 3(a)).

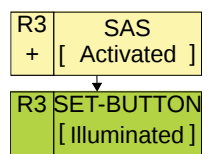

(a) BT3

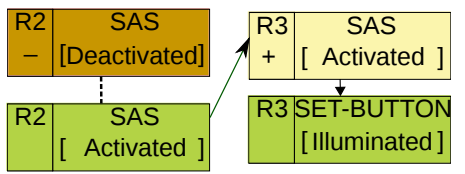

(b) Relation between BT2 \& BT3

Figure 2: Relation in BTs (dots: nodes skipped)
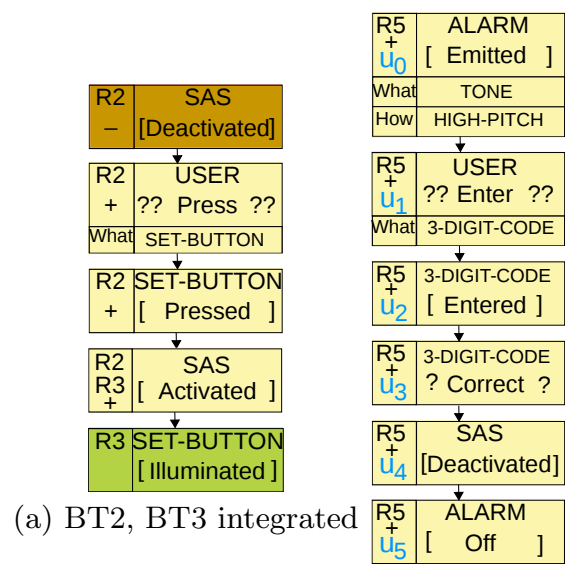

(b) BT5

(a) BT2, BT3 integrated

\section{Figure 3: BT2-3, BT5 and BT6}

We use a very generic representation of Behavioral Model (BM), denoted by $m$, to refer to the mathematical representation of a BT [2]. As such, a BT node is referred to as Behavioral Unit (BU), denoted by $u$. A BU consists of a set of attributes $\left\{a_{k}\right\}$ and so does an edge between two BUs. Each attribute $a_{k}$ has a name, denoted by name $\left(a_{k}\right)$, and a value, denoted by value $\left(a_{k}\right)$. We use parents $(u)$ and childs $(u)$ to denote the set of parent and child BUs.

We say a parent $\mathrm{BM} m_{p}$ forms an integration relation with a child $\mathrm{BM} m_{c}$, denoted by $R\left(m_{p}, m_{c}\right)$, if a BU $u_{p}$ in $m_{p}$ is equivalent to the root BU $u_{c}$ of $m_{c}$ [3, Section-2]. As such, BT5 and BT6 form an integration relation because $u_{2}$ of BT5 and $u_{0}$ of BT6 are equivalent (Fig. 3(b, c)). We use $R^{k}\left(m_{p}, m_{c}\right)$ to denote $k$-th relation between $m_{p}$ and $m_{c}$. We consider three types of integration relation: root-root, branch-root and leaf-root relations [2].

$\operatorname{CSP}_{\sigma}$ : It is an extension of the process algebra CSP. It allows concurrent processes to communicate synchronously

via shared events, and to manipulate and check the value of state variables [4]. A BT is transformed into a $\mathrm{CSP}_{\sigma}$ process, and each node is transformed into a $\mathrm{CSP}_{\sigma}$ action. Different operators control the concurrency in the processes.

SNM: An SNM captures the BMs and their interrelations into an annotated graph, which stores their meta-level information such as their acceptability states (Included (IN), Excluded (EX) and Undecided(UN)), the confidences of the BMs to be Included in the specification and the similarity measure of the parent and child BUs for a relation etc [3].

\section{FORMAL INTEGRATION}

In this section, we first provide the semantics of a BM. We then formalisation the integration of two interrelated BMs.

\subsection{Semantics of a BM: Extension of CSP $_{\sigma}$}

A BM consists of BUs connected by edges. The attributes of each of these entities may have operational semantics. For example, the behavior-type attribute of a BU indicates the type of action such as state realisation, selection, guard, input or output for the component denoted by componentname. The node-operator attribute indicates recursion, interrupt, restart or synchronisation. Thus, a BU represents a $\mathrm{CSP}_{\sigma}$ action characterised by the attributes of the BU. The type-name attribute of an edge indicates atomic, sequential, parallel or alternative step from one action to another. An edge may have timing constraints. Thus, an edge represents the characteristics of the step between actions such as actions performed non-interleaving (atomic), sequential, parallel or external choice (alternative), which may further be executed after a fixed amount of time delay. $\mathrm{CSP}_{\sigma}$ [4] does not consider the edge as an entity, however it provides semantics of atomic, sequential, parallel and alternative steps.

Fig. 4 shows a sample BM having BUs connected with edges. $\mathrm{CSP}_{\sigma}$ defines semantics of the BM considering the attributes (e.g. behavior-type) of the BUs and the type of edges. It also defines nonsequential execution of the processes for node-operator attribute. On top of it, we can extend the $\mathrm{CSP}_{\sigma}$ as follows.

Let $P_{u_{i}}$ denotes the $\mathrm{CSP}_{\sigma}$ process starting from the $\mathrm{CSP}_{\sigma}$ action characterised by

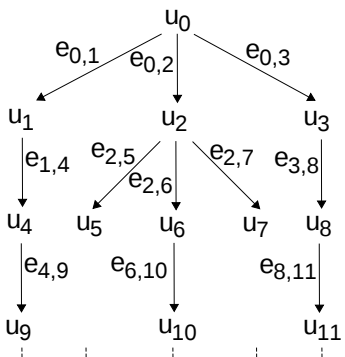

Figure 4: Sample BM with BUs connected with edges the BU $u_{i}$. As such, $P_{u_{0}}$ denotes the $\mathrm{CSP}_{\sigma}$ of the BM shown in Fig. 4. Here, the root BU $u_{0}$ has three children $u_{1}, u_{2}$ and $u_{3}$ connected by $e_{0,1}, e_{0,2}$ and $e_{0,3}$ respectively. If the $\mathrm{CSP}_{\sigma}$ action for $u_{0}$ is performed, the $\mathrm{CSP}_{\sigma}$ process $P_{u_{0}}$ may behave as $P_{u_{1}}, P_{u_{2}}$ and/or $P_{u_{3}}$ after following the steps characterised by the edges $e_{0,1}, e_{0,2}$ and $e_{0,3}$ respectively. Therefore, $P_{u_{0}}$ can be written by the following way:

$$
P_{u_{0}}::=\|_{u_{j}}\left(\left(u_{0}, e_{0, j}\right) \longrightarrow P_{u_{j}}\right) \text { s.t. } u_{j} \in \operatorname{childs}\left(u_{0}\right)
$$

Symbol $\|_{u_{j}}$ indicates concurrent processes for each $u_{j}$.

Definition 1 (Semantics of A BM). The semantics of a BM is a $C S P_{\sigma}$ with BUs as the $C S P_{\sigma}$ actions and edges as the steps from one action to another. Let $P_{u_{i}}$ denotes the $C S P_{\sigma}$ process starting from the action characterised by the $B U u_{i}$, which has children BUs denoted by childs $\left(u_{i}\right)$, then,

$P_{u_{i}}::=\|_{u_{j}}\left(\left(u_{i}, e_{i, j}\right) \longrightarrow P_{u_{j}}\right)$ where $u_{j} \in \operatorname{childs}\left(u_{i}\right)$ 


\subsection{Integrating BMs: Merging $\mathrm{CSP}_{\sigma}$ processes}

If two BMs have root-root, branch-root or leaf-root relation, they can be integrated into one BM by merging their equivalent BUs pair into one BU. By Definition 1, a BM represents a $\mathrm{CSP}_{\sigma}$ process and each $\mathrm{BU}$ represents a $\mathrm{CSP}_{\sigma}$ action. As a result, integrating two BMs means merging two $\mathrm{CSP}_{\sigma}$ processes. This further implies that the $\mathrm{CSP}_{\sigma}$ actions represented by the equivalent $\mathrm{BU}$ can be merged into one $\mathrm{CSP}_{\sigma}$ action. In that case, the attributes that characterise the merged $\mathrm{CSP}_{\sigma}$ action would depend on the merging of the attributes of the equivalent BUs. We refer to the merged $\mathrm{CSP}_{\sigma}$ action as the least common action (lca).

Definition 2 (LEAST COMMON ACTION). If two BMs have an integration relation, the $C S P_{\sigma}$ actions represented by the equivalent $B U s u_{p}$ and $u_{c}$ can be merged into one $C S P_{\sigma}$ action, referred to as the least common action (lca) of the two CSP $P_{\sigma}$ processes represented by the BMs respectively.

In addition, the equivalent BUs may have child BUs which themselves may again be equivalent and so on. That means, the $\mathrm{CSP}_{\sigma}$ actions for the child equivalent $\mathrm{BUs}$ can again be merged into one $\mathrm{CSP}_{\sigma}$ action (Procedure 4); which may continue till a leaf $\mathrm{BU}$ is reached. Thus a refinement of $\mathrm{CSP}_{\sigma}$ processes must follow after the lca to achieve a merged $\mathrm{CSP}_{\sigma}$ process that is more defined than the original two $\mathrm{CSP}_{\sigma}$ processes. In this section, we first illustrate the integration and then formalise it using the extended version of $\mathrm{CSP}_{\sigma}$.

\subsubsection{Illustration of the Integration}

The BU $u_{2}$ in BT5 (Fig. 3(b)) is equivalent to the root BU $u_{0}$ in BT6 (Fig. 3(c)). Therefore, they form an integration relation (Fig. 5(a)). So they can be integrated into one BT. The integration starts from integrating the BUs $u_{2}$ and $u_{0}$ (Fig. 5(b)). Note that the behavior type of these two BUs are different: state realisation and event. Here, state realisation has high precedence over event. So the integrated BU takes state realisation value of the behavior type attribute. After integrating these BUs, the child BUs $u_{3}$ (of BT5) and $u_{1}$ (of BT6) of these former BUs are equivalent again (Fig. 5(b)).

Therefore, the edges $e_{2,3}$ of BT5 and $e_{0,1}$ of BT6 are integrated first and then the BUs $u_{3}$ of BT5 and $u_{1}$ of BT6 are integrated. Now, the child BUs $u_{4}$ (of BT5) and $u_{2}$ (of BT6) further are equivalent (Fig. 5(c)). The integration ends after integrating the edges $e_{3,4}$ of $\mathrm{BT} 5$ and $e_{1,2}$ of BT6, and the BUs $u_{4}$ of BT5 and $u_{2}$ of BT6 (Fig. 5(d)). Thus, the integration starts by integrating the equivalent BUs which cause the integration relation to be formed between the BMs. Then, the integrated BM goes through further refinement by integrating the equivalent child BUs and so on.

\subsubsection{Integration for root-root relation}

Let, $u_{p}$ and $u_{c}$ represent the root BUs of the BMs having root-root relation. From Definition 1 ,

$P_{u_{p}}::=\|_{u_{i}}\left(\left(u_{p}, e_{p, i}\right) \longrightarrow P_{u_{i}}\right)$ where $u_{i} \in \operatorname{childs}\left(u_{p}\right)$

$P_{u_{c}}::=\|_{u_{j}}\left(\left(u_{c}, e_{c, j}\right) \longrightarrow P_{u_{j}}\right)$ where $u_{j} \in \operatorname{childs}\left(u_{c}\right)$

Let, $u_{i}=u_{p}, u_{j}=u_{c}$ as shown in Fig. 6. Suppose $u_{i} \oplus u_{j}$ $\left(=u_{i j}\right)$ denotes their integration. Assume the child BUs $u_{i}^{\prime}$ and $u_{j}^{\prime}$ are again equivalent. Therefore, we have to integrate the edges $\left(e_{i, i^{\prime}} \oplus e_{j, j^{\prime}}=e_{i j, i^{\prime} j^{\prime}}\right)$, and the BUs $\left(u_{i}^{\prime} \oplus u_{j}^{\prime}=u_{i j}^{\prime}\right)$ as indicated by the dotted boxes in Fig. 6. Now,

$$
\begin{array}{r}
P_{u_{i}}::=\left(u_{i}, e_{i, i^{\prime}}\right) \longrightarrow P_{u_{i}^{\prime}} \|_{u_{k}}\left(\left(u_{i}, e_{i, k}\right) \longrightarrow P_{u_{k}}\right) \\
\text { where } u_{k} \in\left\{\operatorname{childs}\left(u_{i}\right)-u_{i}^{\prime}\right\}
\end{array}
$$

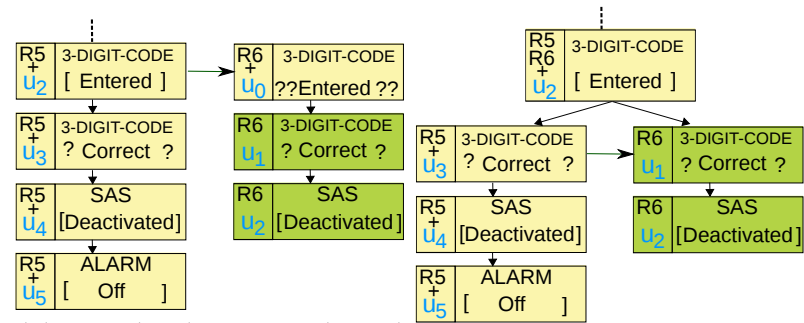

(a) $u_{2}$ (left) \& $u_{0}$ (right) equivalent

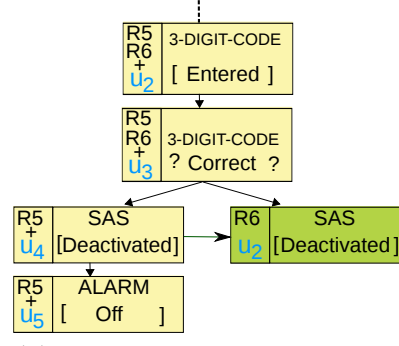

(c) $u_{3} \& u_{1}$ integrated; $u_{4}$ (left) \& $u_{2}$ (right) equivalent

Figure 5: Step-by-step integration of BT5 \& BT6

$$
\begin{array}{r}
P_{u_{j}}::=\left(u_{j}, e_{j, j^{\prime}}\right) \longrightarrow P_{u_{j}^{\prime}} \|_{u_{k}}\left(\left(u_{j}, e_{j, k}\right) \longrightarrow P_{u_{k}}\right) \\
\text { where } u_{k} \in\left\{\operatorname{childs}\left(u_{j}\right)-u_{j}^{\prime}\right\}
\end{array}
$$

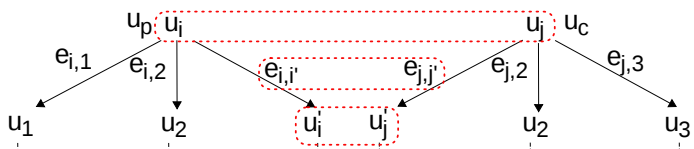

Figure 6: Sample BMs having root-root relations

Let $P_{u_{i}} \oplus P_{u_{j}}::=P_{u_{i j}}$ denotes the merging. Therefore,

$$
\begin{array}{r}
P_{u_{i j}}::=\|_{u_{k}^{\prime}}\left(\left(u_{i j}, e_{i j, i^{\prime} j^{\prime}}, u_{i j}^{\prime}\right), e_{i^{\prime} j^{\prime}, k^{\prime}}\right) \\
\longrightarrow P_{u_{k}^{\prime}} \|_{u_{k}}\left(\left(u_{i j}, e_{i j, k}\right) \longrightarrow P_{u_{k}}\right) \\
\text { where } u_{k}^{\prime} \in\left\{\operatorname{childs}\left(u_{i}^{\prime}\right) \cup \operatorname{childs}\left(u_{j}^{\prime}\right)\right\} \text { and } \\
u_{k} \in\left\{\left(\operatorname{childs}\left(u_{i}\right) \cup \operatorname{childs}\left(u_{j}\right)\right)-\left\{u_{i}^{\prime}, u_{j}^{\prime}\right\}\right\}
\end{array}
$$

Procedure 1 (Integrating root-root Related BMs). If two BMs have root-root relation, the $C S P_{\sigma}$ processes $P_{u_{p}}$ and $P_{u_{c}}$ represented by the $B M s$ can be merged into one $C S P_{\sigma}$ process, denoted by $P_{u_{p c}}::=P_{u_{p}} \oplus P_{u_{c}}$, in two phases:

1) Least Common Action: The merging begins with producing the lca of the two processes. Thus,

$$
\begin{array}{r}
P_{u_{p}} \oplus P_{u_{c}}::=\|_{u_{k}}\left(\left(\left(u_{p} \oplus u_{c}\right), e_{p c, k}\right) \longrightarrow P_{u_{k}}\right) \\
\text { where } u_{k} \in\left\{\operatorname{childs}\left(u_{p}\right) \cup \operatorname{childs}\left(u_{c}\right)\right\}
\end{array}
$$

2) Refinement: Let, $u_{i}=u_{p}, u_{j}=u_{c}$ and so $u_{i j}=u_{p c}$. For each equivalent BUs pairs $u_{i}^{\prime}$ and $u_{j}^{\prime}$ such that $u_{i}^{\prime} \in$ childs $\left(u_{i}\right)$ and $u_{j}^{\prime} \in \operatorname{childs}\left(u_{j}\right)$, the refinement steps are:

(a) Let, we rewrite the CSP $P_{\sigma}$ process $P_{u_{p c}}$ as $P_{u_{p c}}::=P_{u_{p c}} \leadsto$ $P_{u_{i j}}$ denoting $P_{u_{p c}}$ contains a CSP $P_{\sigma}$ process $P_{u_{i j}}$ at a certain point in time along with other concurrent processes. Here,

$$
\begin{aligned}
P_{u_{i j}}::= & \|_{u_{k}^{\prime}}\left(\left(\left(u_{i} \oplus u_{j}\right),\left(e_{i, i^{\prime}} \oplus e_{j, j^{\prime}}\right),\left(u_{i}^{\prime} \oplus u_{j}^{\prime}\right)\right),\right. \\
& \left.e_{i^{\prime} j^{\prime}, k^{\prime}}\right) \longrightarrow P_{u_{k}^{\prime}} \|_{u_{k}}\left(\left(u_{i j}, e_{i j, k}\right) \longrightarrow P_{u_{k}}\right) \\
& \text { where } u_{k}^{\prime} \in\left\{\operatorname{childs}\left(u_{i}^{\prime}\right) \cup \operatorname{childs}\left(u_{j}^{\prime}\right)\right\} \text { and } \\
& u_{k} \in\left\{\left(\operatorname{childs}\left(u_{i}\right) \cup \operatorname{childs}\left(u_{j}\right)\right)-\left\{u_{i}^{\prime}, u_{j}^{\prime}\right\}\right\}
\end{aligned}
$$

(b) Assign $u_{i}=u_{i}^{\prime}$ and $u_{j}=u_{j}^{\prime}$, and perform Refinement. 


\subsubsection{Integration for branch-root relation}

Let, $u_{p}$ and $u_{c}$ represent the equivalent BUs of the BMs having branch-root relation. Suppose, $u_{r}$ denotes the root of the parent BM. Let, we rewrite the $\mathrm{CSP}_{\sigma}$ process $P_{u_{r}}$ as $P_{u_{r}}::=P_{u_{r}} \leadsto P_{u_{p}}$. Let, $u_{i}=u_{p}, u_{j}=u_{c}$ as shown in Fig. 7. The equivalent BUs and corresponding edges have to be integrated as indicated by the dotted boxes in Fig. 7 . $P_{u_{i j}}$ of $P_{u_{i}}$ and $P_{u_{j}}$ can be formed similarly by Equation 7 .

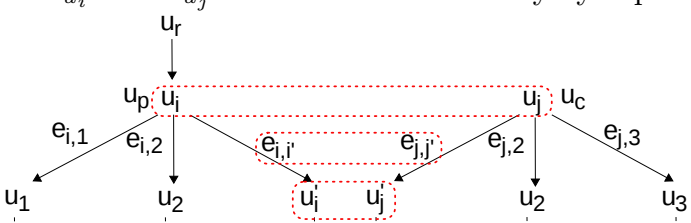

Figure 7: Sample BMs having branch-root relations

Procedure 2 (INTEGRATING branch-root RELATED BMs). If two BMs have branch-root relation, the CSP $P_{\sigma}$ processes $P_{u_{r}}$ and $P_{u_{c}}$ represented by the BMs can be merged into one $C S P_{\sigma}$ process, denoted by $P_{u_{r}}::=P_{u_{r}} \oplus P_{u_{c}}$, in two phases:

1) Least Common Action: The merging begins with producing the lca of the two processes $P_{u_{p}}$ and $P_{u_{c}}$. Thus,

$$
\begin{array}{r}
P_{u_{r}}::=P_{u_{r}} \leadsto P_{u_{p c}} \text { where } \\
P_{u_{p c}}::=\|_{u_{k}}\left(\left(\left(u_{p} \oplus u_{c}\right), e_{p c, k}\right) \longrightarrow P_{u_{k}}\right) \\
\text { where } u_{k} \in\left\{\operatorname{childs}\left(u_{p}\right) \cup \operatorname{childs}\left(u_{c}\right)\right\}
\end{array}
$$

2) Refinement: Let, $u_{i}=u_{p}, u_{j}=u_{c}$ and so $u_{i j}=u_{p c}$. For each equivalent BUs pairs $u_{i}^{\prime}$ and $u_{j}^{\prime}$ such that $u_{i}^{\prime} \in \operatorname{childs}\left(u_{i}\right)$ and $u_{j}^{\prime} \in \operatorname{childs}\left(u_{j}\right)$, the refinement steps are:

(a) Let, we rewrite $P_{u_{r}}$ as $P_{u_{r}}::=P_{u_{r}} \leadsto P_{u_{i j}}$. Here,

$$
\begin{array}{r}
P_{u_{i j}}::=\|_{u_{k}^{\prime}}\left(\left(\left(u_{i} \oplus u_{j}\right),\left(e_{i, i^{\prime}} \oplus e_{j, j^{\prime}}\right),\left(u_{i}^{\prime} \oplus u_{j}^{\prime}\right)\right),\right. \\
\left.e_{i^{\prime} j^{\prime}, k^{\prime}}\right) \longrightarrow P_{u_{k}^{\prime}} \|_{u_{k}}\left(\left(u_{i j}, e_{i j, k}\right) \longrightarrow P_{u_{k}}\right) \\
\text { where } u_{k}^{\prime} \in\left\{\operatorname{childs}\left(u_{i}^{\prime}\right) \cup \operatorname{childs}\left(u_{j}^{\prime}\right)\right\} \\
\text { and } u_{k} \in\left\{\left(\operatorname{childs}\left(u_{i}\right) \cup \operatorname{childs}\left(u_{j}\right)\right)-\left\{u_{i}^{\prime}, u_{j}^{\prime}\right\}\right\}
\end{array}
$$

(b) Assign $u_{i}=u_{i}^{\prime}$ and $u_{j}=u_{j}^{\prime}$, and perform Refinement.

\subsubsection{Integration for leaf-root relation}

Let, $u_{p}$ and $u_{c}$ represent the equivalent BUs of the BMs having leaf-root relation. Suppose, $u_{r}$ denotes the root of the parent BM. Since it is a leaf-root relation, there exists no child of the leaf BU. Let, we rewrite the $\operatorname{CSP}_{\sigma}$ process $P_{u_{r}}$ as $P_{u_{r}}::=P_{u_{r}} \leadsto P_{u_{p}}$. Fig. 8 shows that the merging is performed by integrating the equivalent BUs $u_{p}$ and $u_{c}$.

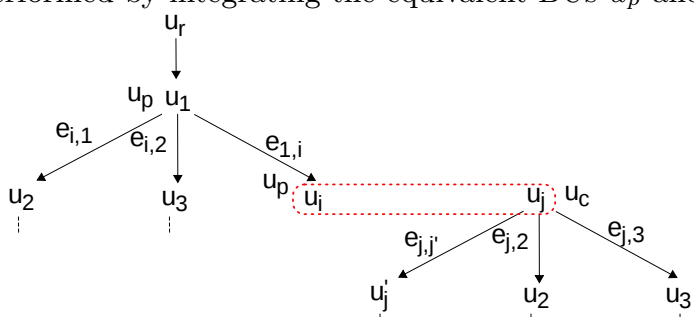

Figure 8: Sample BMs having leaf-root relations

Procedure 3 (Integrating leaf-root Related BMs).

If two BMs have leaf-root relation, the corresponding processes $P_{u_{r}}$ and $P_{u_{c}}$ can be merged into one CSP $P_{\sigma}$ process, denoted by $P_{u_{r}}::=P_{u_{r}} \oplus P_{u_{c}}$, by producing the lca:

$$
\begin{array}{r}
P_{u_{r}}::=P_{u_{r}} \leadsto P_{u_{p c}} \text { where } \\
P_{u_{p c}}::=\|_{u_{k}}\left(\left(\left(u_{p} \oplus u_{c}\right), e_{p c, k}\right) \longrightarrow P_{u_{k}}\right) \\
\text { where } u_{k} \in \operatorname{childs}\left(u_{c}\right)
\end{array}
$$

\subsection{Integrating BUs: Merging CSP $_{\sigma}$ Actions}

If two BUs are equivalent, the integration of them represents a merged $\mathrm{CSP}_{\sigma}$ action. It is characterised by the attributes of the integrated $\mathrm{BU}$, which we get by integrating the attributes of the given BUs.The integration of two attributes $a_{i}$ and $a_{j}$, given their names are same, is denoted by $a_{i} \oplus a_{j}$; which is formalised in Section 3.4. The names of the attributes may not be syntactically same, but may have same mapping. We assume that names of the attributes are syntactically same if they have the same mapping.

Procedure 4 (Integrating BUs: $u_{p} \oplus u_{c}$ ). If the $B U s$ $u_{p}$ and $u_{c}$ are equivalent, the corresponding $C S P_{\sigma}$ actions can be merged into one $C S P_{\sigma}$ action that is characterised by the attributes of the $B U u_{p c}=u_{p} \oplus u_{c}$ formed by integrating the attributes of $u_{p}$ and $u_{c}$ by the following rules:

1) If there exists a pair of attributes $a_{i} \in u_{p}$ and $a_{j} \in u_{c}$ such that name $\left(a_{i}\right)=$ name $\left(a_{j}\right)$, then an attribute $a_{k} \in u_{p c}$ is formed such that $a_{k}=a_{i} \oplus a_{j}$.

2) If there exists an attribute $a_{i} \in u_{p}$ such that name $\left(a_{i}\right) \neq$ name $\left(a_{j}\right)$ for all attribute $a_{j} \in u_{c}$, then an attribute $a_{k} \in$ $u_{p c}$ is formed such that $a_{k}=a_{i}$.

3) If there exists an attribute $a_{j} \in u_{c}$ such that name $\left(a_{j}\right) \neq$ name $\left(a_{i}\right)$ for all attribute $a_{i} \in u_{p}$, then an attribute $a_{k} \in u_{p c}$ is formed such that $a_{k}=a_{j}$.

Due to similarity we omit the formalisation of integrating edges i.e. merging the steps between the $\mathrm{CSP}_{\sigma}$ actions.

\subsection{Integrating attributes}

When integrating two BUs or edges, we essentially integrate the attributes of these entities. If the names of the attributes $a_{i}$ and $a_{j}$ are same, we form an integrated attribute $a_{k}=a_{i} \oplus a_{j}$. The value of the integrated attribute $a_{k}$ depends on the values of the attributes $a_{i}$ and $a_{j}$. If the values are same, then the integrated value is also the same. Otherwise, the value of the integrated attribute is formed by many different operations between the original values.

Procedure 5 (Integrating attributes: $a_{i} \oplus a_{j}$ ). If two attributes $a_{i}$ and $a_{j}$, given that name $\left(a_{i}\right)=$ name $\left(a_{j}\right)$, are integrated into one attribute $a_{k}$ where name $\left(a_{k}\right)=$ name $\left(a_{i}\right)$, then value $\left(a_{k}\right)$ is formed by the following rules:

1) If value $\left(a_{i}\right)=\operatorname{value}\left(a_{j}\right)$, then value $\left(a_{k}\right)=\operatorname{value}\left(a_{i}\right)$.

2) Otherwise, value $\left(a_{k}\right)=\operatorname{value}\left(a_{i}\right) \oplus \operatorname{value}\left(a_{j}\right)$.

The integration of the values i.e. value $\left(a_{i}\right) \oplus \operatorname{value}\left(a_{j}\right)$ can be performed in many different ways.

Choice: The value of the integrated attribute may be selected from the values of the original values. For example, the values of the behavior type attribute may be different. One may have state realisation ([]) and other may have selection (??) as values. In this case, the value of the integrated attribute would be state realisation. In this case, we say that the property of the attribute behavior type is choice.

If the property of an attribute is choice, the values of that attribute are kept into a sorted set from high to low precedence order. For example, state realisation has a high precedence over selection for the behavior type attribute.

Combination: The value of the integrated attribute may be the combination of the original values. For example, the value of traceability link attribute may be different. The values may be $R 2$ and $R 3$. In this case, the value of the integrated attribute would be the combination of those values i.e. $\{\mathrm{R} 5, \mathrm{R} 6\}$. We say that the property of the attribute traceability link is combination. 
Mapping: The values of the attributes may be syntactically different, but semantically same. For example, the component name of a component may have aliases, but all these aliases refer to the same component.

Thus, the integration of the values may be the mapped value from the original values, intersection of the values, mathematical operation and so on. Which strategy should be taken depends on the domain information.

\subsection{Theorems and Properties}

Corollary 1 (Closure of Merging). (1) The merging of the two CSP $\sigma$ actions by Procedure 4 produces another $\mathrm{CSP}_{\sigma}$ action and (2) the integration of two attributes by Procedure 5 produces another attribute.

Theorem 1 (Preservation of Semantics). The semantics of the integrated BM by Procedures 1, 2 and 3 is consistent with the semantics of the given two input BMs.

Theorem 2 (Polynomial Complexity). Given two $B M s$ having edge sets $E_{1}$ and $E_{2}$, the worst-case complexity of (1) Procedure 1 is $O\left(\min \left(e_{1}, e_{2}\right)\right)$, (2) Procedure 2 is $O\left(e_{2}\right)$ and (3) Procedure 3 is $O(1), e_{1}=\left|E_{1}\right|, e_{2}=\left|E_{2}\right|$.

Theorem 3 (Closure of Merging CSP $\sigma$ Processes). The merging of the two CSP $\sigma$ processes by Procedures 1, 2 and 3 also produce a $C S P_{\sigma}$ process.

Theorem 4 (Optimal Refinement). Procedures 1, 2 and 3 produce an optimally refined $\mathrm{CSP}_{\sigma}$ process from the given two $C S P_{\sigma}$ processes.

Properties of the merging of $\mathbf{C S P}_{\sigma}$ processes: We get the following properties:

1) Reflexive: $P_{u_{i}} \oplus P_{u_{i}}=P_{u_{i}}$. 2) Asymmetry: $P_{u_{i}} \oplus P_{u_{j}} \neq$ $P_{u_{j}} \oplus P_{u_{i}}$. 3) Partial Symmetry: $P_{u_{i}} \oplus P_{u_{j}}=P_{u_{j}} \oplus P_{u_{i}}$ if the corresponding BMs have root-root relation. 4) Associative: $P_{u_{i}} \oplus P_{u_{j}} \oplus P_{u_{k}}=\left(P_{u_{i}} \oplus P_{u_{j}}\right) \oplus P_{u_{k}}=P_{u_{i}} \oplus\left(P_{u_{j}} \oplus P_{u_{k}}\right)$.

Properties of the merging of $\operatorname{CSP}_{\sigma}$ actions, edges and attributes: Each operation shares the same set of properties. We mention the properties for $\operatorname{CSP}_{\sigma}$ actions:

1) Reflexive: $u_{i} \oplus u_{i}=u_{i}$. 2) Symmetric: $u_{i} \oplus u_{j}=u_{j} \oplus u_{i}$. 3) Associative: $u_{i} \oplus u_{j} \oplus u_{k}=\left(u_{i} \oplus u_{j}\right) \oplus u_{k}=u_{i} \oplus\left(u_{j} \oplus u_{k}\right)$.

Proofs will be available in the longer version of the paper.

\section{INTEGRATION OF A SET OF BMS}

In this section, we use an SNM of the interrelated BMs and develop an algorithm to integrate all the BMs into one BM. Let, $m_{1}$ to $m_{7}$ denote the BMs of the SAS. Suppose they are interrelated to each other as shown in Table 1. The column BMs shows the pair of parent and child BMs, Relations $\left(u_{p}, \odot\right)$ shows the type of relation, parent BU $\left(u_{p}\right)$ and the similarity measure $(\odot)$ of the parent and child BUs.

Table 1: Relations of the BMs of the SAS.

\begin{tabular}{|c|c|c|c|}
\hline BMs & Relations $\left(u_{p}, \odot\right)$ & BMs & Relations $\left(u_{p}, \odot\right)$ \\
\hline$m_{2}, m_{3}$ & leaf-root $\left(u_{3}, 1\right)$ & $m_{2}, m_{4}$ & leaf-root $\left(u_{3}, 1\right)$ \\
\hline$m_{3}, m_{4}$ & $\operatorname{root}-\operatorname{root}\left(u_{0}, 1\right)$ & $m_{4}, m_{3}$ & $\operatorname{root-root}\left(u_{0}, 1\right)$ \\
\hline$m_{4}, m_{5}$ & leaf-root $\left(u_{2}, 1\right)$ & $m_{6}, m_{2}$ & leaf-root $\left(u_{2}, 1\right)$ \\
\hline$m_{5}, m_{6}$ & branch-root $\left(u_{2}, .75\right)$ & $m_{5}, m_{7}$ & branch-root $\left(u_{2}, .75\right)$ \\
\hline$m_{6}, m_{7}$ & 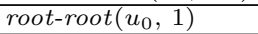 & $m_{7}, m_{6}$ & 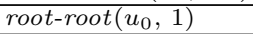 \\
\hline$m_{3}, m_{1}$ & $\operatorname{root-\operatorname {root}(u_{0},1)}$ & $m_{1}, m_{3}$ & root-root $\left(u_{0}, 1\right)$ \\
\hline$m_{4}, m_{1}$ & $\operatorname{root}-\operatorname{root}\left(u_{0}, 1\right)$ & $m_{1}, m_{4}$ & $\operatorname{root-\operatorname {root}(u_{0},1)}$ \\
\hline$m_{2}, m_{1}$ & leaf-root $\left(u_{3}, 1\right)$ & $m_{5}, m_{2}$ & branch-root $\left(u_{4}, 1\right)$ \\
\hline
\end{tabular}

Fig. 9 shows a sample SNM created from the Table 1. We assumed that $m_{2}$ is the initialisation (Init) model. Initially, $m_{2}$ is IN, and all other BMs are EX. For a BM, we assign confidence 1 if a BM is IN and 0 if EX. Otherwise, confidence of a BM is the confidence of its parent multiplied by the similarity measure of its corresponding integration relation. Further, a relation is annotated by its acceptability state. We consider two layers of thresholds namely (1) IN threshold $\alpha_{\text {IN }}$ and (2) UN threshold $\alpha_{\text {UN }}$ where $\alpha_{\text {UN }}<\alpha_{\text {IN }}$. We assume a relation is IN if similarity $\geq \alpha_{\mathrm{IN}}$, and EX if similarity $<\alpha_{\mathrm{UN}}$, and UN otherwise. Assume that $\alpha_{\mathbb{I N}}=1$ and $\alpha_{\mathrm{UN}}=0.75$.

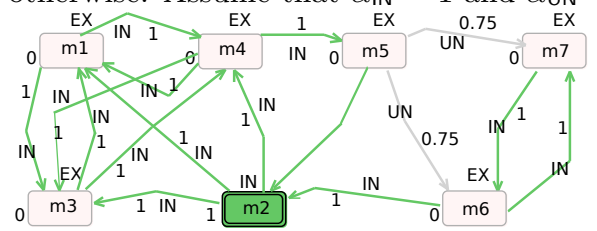

Figure 9: SNM showing initial states, relation equivalences and confidence levels

The acceptability states of the other BMs can be inferred. For example, $m_{2}$ is IN and the relation between $m_{2} \& m_{3}$ is also IN. Therefore, $m_{3}$ can be inferred as IN. We infer $\mathrm{UN}(m)$ if it is not IN and there exists at least one pair of parent and relation such that both are not EX. We infer $\operatorname{EX}(m)$ if it is neither IN nor UN. Further, The SNM shown in Fig. 9 can be made well-formed by eliminating redundant relations, breaking cycles and assigning the states as shown in Fig. 10. Assume that the relations between $m_{5} \& m_{6}$ and $m_{5} \& m_{7}$ have been accepted by the requirements analyst. Informally, we say an SNM is well-formed, if (1) the SNM is a directed acyclic graph, (2) the number of Init models in the SNM is 1, (3) all models are IN, and (4) all existing relations are IN [3]. We use childs $\left(m_{i}\right)$ and parents $\left(m_{i}\right)$ to denote child and parent BMs respectively.

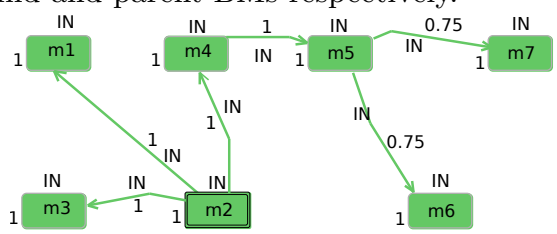

Figure 10: well-formed SNM

We can traverse the well-formed SNM from the Init model by breadth first search, and integrate the BMs into one BM.

Procedure 6 (Integrating BMs). Given a well-formed $S N M G=\langle\mathcal{M}, \mathcal{R}\rangle$ where $\mathcal{M}$ is the set of BMs and $\mathcal{R}$ is the set of relations in the well-formed $S N M$, the integration of the BMs are performed by Algorithm 1.

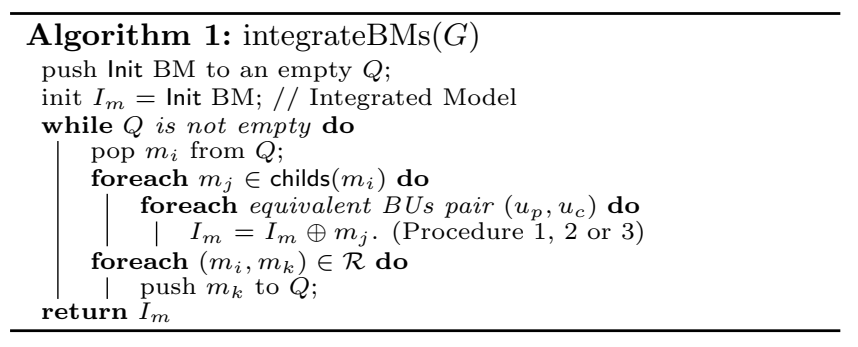

Theorem 5 (Polynomial Complexity). Given relations $\mathcal{R}$ in the well-formed $S N M G$, the worst-case complexity of Algorithm 1 (integrateBMs) is $O(r), r=|\mathcal{R}|$.

Theorem 6 (Soundness \& Completeness). (1) Given $a$ well-formed SNM, Procedure 6 produces an integrated BM in polynomial time, (2) Procedure 6 can be applied to any well-formed SNM to produce an integrated BM. 


\section{RELATED WORK}

We classify the related work into two categories: Formalisation of BE, and Integrating Software Models.

Formalisation of BE: Colvin and Hayes [4] provided the semantics of the BT using $\mathrm{CSP}_{\sigma}$. We extended the semantics by including the edge between nodes as an entity in $\mathrm{CSP}_{\sigma}$. Winter et al. [24] developed a framework of rules for integrating requirements using a notation-independent graphical model. Though they discussed overall broad approach of BTs integration, the rules do not relate the integration with BT's semantics. They also did not develop the techniques to automatically integrate a set of BTs.

BT has been used for model-checking to assess the safety requirements [12], analyse role-based access control [26], perform Failure Modes and Effects Analysis (FMEA) [8], to identify the combinations of component failures by Cut Set Analysis (CSA) [11], verify large systems using a slicing techniques [25] and so on. Myers et al. [16] simulated behavioral scenarios using BTs into Modelica framework.

Integrating Software Models: In earlier stage ([9], [13]), the main focus was to do parallel composition of models that explains how two different components work together. Since different UML diagrams have different syntax and semantics, merging of those models are mostly manual ([7], [22]). Uchitel and Chechik [21] worked on merging two partial behavioral models of the same component to produce an elaborated version of both original models. Our works differ from these works in composing two behavioral models depicting state-based and scenario-based behavioral scenarios of more than one interacting components of the software system, which may have overlapping system behaviors, to obtain a more compact version of the original two.

\section{CONCLUSION}

In this paper, we provided a formal definition of the integration of the BTs. We first formalised the semantic integration of two interrelated BTs using an extended version of $\mathrm{CSP}_{\sigma}$. We then formalised the integration of a set of BTs. Our future research focus is to formalise specification process where we would like to semi-automate the tasks to convert an integrated BT into a specification BT which can be validated by simulation and verified by model-checking.

\section{REFERENCES}

[1] Raytheon Australia. www.raytheon.com.au

[2] Ahmed, K., Myers, T., Wen, L., Sattar, A.: Detecting Requirements Defects Utilizing A Mathematical Framework for Behavior Engineering. JSCSE, Advanced Academic Publisher 3(3), 187-198 (2013)

[3] Ahmed, K., Wen, L., Sattar, A.: iRE: A Semantic Network based Interactive RE Framework. In: 2nd World Conference on Complex Systems (Nov 2014)

[4] Colvin, R.J., Hayes, I.J.: A Semantics for BTs Using CSP with Specification Commands. Science of Computer Programming 76(10), 891 - 914 (2011)

[5] Dromey, R.G.: Formalizing the Transition from Requirements to Design. Mathematical Frameworks for Component Software-Models for Analysis and Synthesis pp. 156-187 (2006)

[6] Eugster, P.T., Felber, P.A., Guerraoui, R., Kermarrec, A.M.: The many faces of publish/subscribe. ACM Comput. Surv. 35(2), 114-131 (Jun 2003)
[7] Fortsch, S., Westfechtel, B.: Differencing and Merging of Software Diagrams - State of the Art and Challenges. ICSOFT (2007)

[8] Grunske, L., Winter, K., Yatapanage, N., Zafar, S., Lindsay, P.A.: Experience with Fault Injection Experiments for FMEA. Software: Practice and Experience 41(11), 1233-1258 (2011)

[9] Hoare, C.A.R.: Communicating Sequential Processes. Series in Computer Science (1985)

[10] Lee, P., Tran, T., Phillips, V., Papacostantinou, P., Aitken, A., Rosbotham, S.: Implementing a behaviour tree analysis tool using eclipse development frameworks. In: 19th ASWEC 2008. p. 61 (2008)

[11] Lindsay, P., Yatapanage, N., Winter, K.: Cut Set Analysis Using Behavior Trees and Model Checking. Formal Aspects of Computing 24, 249-266

[12] Lindsay, P., Winter, K., Yatapanage, N.: Safety Assessment Using BTs and Model Checking. In: 8th IEEE SEFM. pp. 181 -190 (Sept 2010)

[13] Milner, R.: Communication and concurrency. Prentice-Hall, Inc., NJ, USA (1989)

[14] Myers, T.: TextBE: A Textual Editor for Behavior Engineering. 3rd Improving Systems and Software Engineering Conference (ISSEC) (2-5 August 2011)

[15] Myers, T.: The Foundations for a Scaleable Methodology for Systems Design (PhD Thesis, School of ICT, Griffith University, Australia, 2011)

[16] Myers, T., Dromey, R.G., Fritzson, P.: Comodeling: from Req. to an Integrated Software-Hardware Model. IEEE Computer 44(4), 62 -70 (April 2011)

[17] Prowell, S.J., Trammell, C.J., Linger, R.C., Poore, J.H.: Cleanroom Software Engineering: Technology and Process. Addison-Wesley, MA, USA (1999)

[18] Rest, O., Wachsmuth, G., Steel, J., Sub, J., Visser, E.: Robust real-time synchronization between textual and graphical editors. In: Theory and Practice of Model Transformations, LNCS, vol. 7909 (2013)

[19] Roscoe, A.W.: The Theory and Practice of Concurrency. Series in Computer Science (1998)

[20] Smith, C., Winter, K., Hayes, I., Dromey, R.G., Lindsay, P., Carrington, D.: An Environment for Building A System out of Its Requirements. In: 19th ASE. pp. 398-399 (Sep 2004)

[21] Uchitel, S., Chechik, M.: Merging partial behavioural models. SIGSOFT S/E Notes 29(6), 43-52 (Oct 2004)

[22] Welle, D., Kelter, U.: Merging UML Documents. Internal Report, University of Siegen (2004)

[23] Wen, L., Colvin, R., Lin, K., Seagrott, J., Yatapanage, N., Dromey, R.G.: 'Integrare', a Collaborative Environment for Behavior-oriented Design. In: Luo, Y. (ed.) Cooperative Design, Visualization, and Engg., LNCS, vol. 4674, pp. 122-131. Springer (2007)

[24] Winter, K., Hayes, I.J., Colvin, R.: Integrating Requirements: The Behavior Tree Philosophy. In: 8th IEEE SEFM. pp. 41 -50 (sept 2010)

[25] Yatapanage, N., Winter, K., Zafar, S.: Slicing BT Models for Verification. In: Theoretical Computer Science, vol. 323, pp. 125-139. Springer (2010)

[26] Zafar, S., Dromey, R.G.: Integrating Safety and Security Requirements into Design of An Embedded System. In: 12th APSEC. p. 8 pp. (dec 2005) 